\title{
Weighted Evolving Networks
}

\author{
S.H. Yook, H. Jeong, A.-L. Barabási \\ Department of Physics, University of Notre Dame, Notre Dame, IN 46556 \\ Y. Tu \\ IBM, TJ Watson Research Center, POB 218, Yorktown Heights, NY 10598 USA
}

(October 29, 2018)

\begin{abstract}
Many biological, ecological and economic systems are best described by weighted networks, as the nodes interact with each other with varying strength. However, most network models studied so far are binary, the link strength being either 0 or 1 . In this paper we introduce and investigate the scaling properties of a class of models which assign weights to the links as the network evolves. The combined numerical and analytical approach indicates that asymptotically the total weight distribution converges to the scaling behavior of the connectivity distribution, but this convergence is hampered by strong logarithmic corrections.
\end{abstract}

PACS number: 84.35.+i, 05.40.-a, 02.50.Cw, 87.23.Ge

With the increased availability of detailed topological data on complex networks describing communication, social or biological systems emerges the need to develop tools to understand in general terms the origin and evolution of these complex evolving systems. The recognition that real networks are fundamentally different from the random models that dominated the mathematical literature in the past forty years [1.2] lead to a surge of activity in addressing the statistical properties of these systems [3 10]. Despite rapid advances in uncovering the topology of complex networks, in one aspect most models are incomplete when compared with real systems: current network models assume that all links are equivalent. But in many fields it is well known that the interaction strengths can vary widely, such variations being essential to the network's ability to carry on its basic functions. Sociologists have repeatedly argued about the importance of assigning strengths to social links, finding that the weak links people have outside their close circle of friends play a key role in keeping the social system together 11. Recently, Newman has showed that assigning weights to the links between scientists allows for a better characterization of the scientific collaboration web [12]. Similarly, there is an ongoing discussion about the importance of weak links between species in guaranteeing the stability of an ecosystem [13]; in addition viewing the economy as a complex network of companies, the monetary value of the various business transactions linking them is a key component of the systemic characterization. Finally, many transportation networks nature designed, ranging from cardiovascular to respiratory networks, have well defined weights or flow rates assigned to the links, whose magnitude is intimately determined by the network's topology [14]. Despite the known importance of interaction strengths in various well known systems, there have been no attempts to model networks other than binary nets, whose links have weights 0 or 1 .

In this paper we take a first step in the direction of a systematic study of networks with non-binary connectivities. We introduce and investigate two models that assign weights to new links as they are dynamically created, providing a prototype of a weighted evolving network. While we choose the simplest possible models, in which the weights are driven by the network connectivity only, numerical simulations indicate that the distribution of the total weight scales differently from the total connectivity. However, an analytical solution reveals that the different scaling behavior can be explained by strong logarithmic correction, and asymptotically the investigated weighted networks belong to the same universality class as their unweighted counterparts.

Weighted Scale-Free (WSF) Model : Starting from a small number $\left(m_{0}\right)$ of vertices, at each time step we add a new node which links to $m$ existing nodes in the system. The probability that a new node $j$ will connect to a existing node $i$ is

$$
\Pi_{i}=\frac{k_{i}}{\sum_{j} k_{j}},
$$

where $k_{i}$ is the total number of links that the node $i$ has. In assigning a weight to the newly established link $j \leftrightarrow i$, we assume that the weight $w_{j i}\left(=w_{i j}\right)$ is proportional to $k_{i}$, i.e., more connected (and therefore more "powerful") nodes gain more weight. Also, one can assume that all new nodes have fairly uniform total 'resources' for linking to other nodes in the system, we therefore require that each new node has a fixed total weight, i.e. we normalize $w_{i j}$ such that the sum of the weights for the $m$ new links is $\sum_{\left\{i^{\prime}\right\}} w_{j i^{\prime}}=1$, where $\left\{i^{\prime}\right\}$ represents a sum over the $m$ existing nodes to which the new node $j$ is connected. As a result of the two assumptions, each link $i \leftrightarrow j$ of the newly added node $j$ is assigned a weight as

$$
w_{j i}=\frac{k_{i}}{\sum_{\left\{i^{\prime}\right\}} k_{i^{\prime}}} .
$$


Weighted Exponential (WE) Model: The model is inspired by model A discussed in Refs. [15, 16], and is defined as follows : at every time step we add a new node with $m\left(\leq m_{0}\right)$ links, connected with equal probability to the nodes present in the system. The weights of the links are assigned again by using (2).

The difference between the WSF and WE models comes in preferential attachment, which is known to fundamentally alter the topology $17,9,15-17]$ : The WSF model generates a scale-free network whose connectivity distribution follows $P(k) \sim k^{-3}$, while the network generated by the WE model is exponential with the connectivity distribution following $P(k)=\frac{e}{m} e^{-k / m}$. Since the weights of the links are driven by the connectivity, this difference is expected to lead to significant changes in the distribution of the link strengths as well.

We start by investigating the weight distribution of the two models. As Fig 1 (a) and (b) shows, both the WE and the WSF models lead to a peaked and skewed weight distribution, whose tails decay exponentially (or faster) for large $w_{i j}$. The boundedness of $P\left(w_{i j}\right)$ is due to the normalization condition, which does not allow individual weights to be larger than 1 . Most important, however, we find that the distribution is stationary, i.e. $P\left(w_{i j}\right)$ is independent of time (and system size).

While the individual weights assigned to links, $w_{i j}$, are bounded, we get a very different picture when we study the total weight associated with a selected node. In binary networks the node's importance is characterized by the total number of links it has, $k_{i}$. Similarly, in a weighted network the importance of a node $i$ can be measured by its total weight, obtained by summing the weights of the links that connect to it, $w_{i}=\sum_{\{j\}} w_{i j}$.

Due to the normalization condition (2) a new node has $w_{i}=1$, but $w_{i}$ increases in time every time when a subsequently added nodes link to $i$. Since in both models the weights are determined by the network connectivity, we expect that $P(w)$ closely follows $P(k)$. In contrast, the numerical results summarized in Fig. 2 indicate striking differences between $P(k)$ and $P(w)$. As Fig 2a shows, while for the WE model $P(k)$ decays exponentially, $P(w)$ systematically deviates from a simple exponential behavior. This difference is even more evident in the network dynamics: while both $k_{i}(t)$ and $w_{i}(t)$ appear to increase logarithmically in time, they can be fitted with a different slope on a log-linear plot (Fig. 2b). Similar systematic discrepancies are observed for the WSF model as well: as Fig. 2c indicates, while $P(w)$ can be fitted by a power law, $P(w) \sim w^{-\sigma}$, it appears that the exponent $\sigma$ is different from $\gamma=3$. Furthermore, we find that $\sigma$ depends strongly on $m$ (Fig. 2c). Again, this difference is reflected in the dynamical behavior of $k_{i}(t)$ and $w_{i}(t)$ : as Fig. 2e indicates, $w_{i}(t) \sim t^{\beta}$ with $\beta>1 / 2$, in contrast with $k_{i}(t) \sim t^{1 / 2}[6,16]$ predicted by the binary scale-free model.

To understand the different behaviors of $w_{i}$ and $k_{i}$ uncovered by the numerical simulations, we resort to ana- lytical method in determining the averaged behavior of $w_{i}(t)$ for the discussed model. To simplify the discussion in the following we assume $m=2$, however, the calculations can be generalized for arbitrary $m$. The total weight of node $i$ at time $t$ can be written as

$$
w_{i}(t)=1+\sum_{\{j\}} w_{i j}=1+\int_{t_{i}^{0}}^{t} \tilde{P}_{i}\left(t^{\prime}\right)\left\langle w_{i j}\left(t^{\prime}\right)\right\rangle d t^{\prime},
$$

where $\tilde{P}_{i}(t)$ is the probability that node $i$ is selected to be connected to a new node $j$ at time $t$ and $t_{i}^{0}$ is the time at which the node $i$ has been added to the system. $\left\langle w_{i j}\right\rangle$ is the average weight of link $i \leftrightarrow j$ once the link is established. When a new node $j$ and the list of $m$ nodes $\left\{i^{\prime}\right\}$ to which it connects are selected, the weights of the links, $w_{j i^{\prime}}$ are assigned according to (2). These weights depend on the number of links the selected nodes have, i.e. $\left\{k_{i^{\prime}}\right\}$. If we assume that node $j$ is connected to nodes $i$ and $l(m=2)$, we have

$$
\left\langle w_{i j}(t)\right\rangle=\int_{m}^{\infty} w_{j i}(l) \mathcal{P}\left(k_{l}\right) d k_{l}
$$

where $w_{j i}(l)$ is the weight between the $j$ and $i$ nodes, $\mathcal{P}\left(k_{l}\right)$ is the probability distribution of $k_{l}$, the total link number of node $l$. Substituting (4) into (3), we obtain

$$
w_{i}(t)=1+\int_{t_{i}^{0}}^{t} \int_{m}^{\infty} \tilde{P}_{i}\left(t^{\prime}\right) w_{j i}(l) \mathcal{P}\left(k_{l}\right) d k_{l} d t^{\prime} .
$$

According to (2) for $m=2$, the weight $w_{j i}(l)$ is given by

$$
w_{j i}(l)=\frac{k_{i}}{k_{i}+k_{l}},
$$

thus Eq.([D) becomes

$$
w_{i}(t)=1+\int_{t_{i}^{0}}^{t} \int_{m}^{\infty} \tilde{P}_{i}\left(t^{\prime}\right) \frac{k_{i}}{k_{i}+k_{l}} \mathcal{P}\left(k_{l}\right) d k_{l} d t^{\prime} .
$$

Eq. (7) represents a general expression for calculating $w_{i}(t)$ for $m=2$. To apply it to the WE and WSF models, we need to calculate explicitly $\tilde{P}(t)$ and $\mathcal{P}\left(k_{l}\right)$.

WE model: In the WE model the nodes to which a new node connects to are selected uniformly among all existing nodes, thus the probability that node $i$ will be picked is independent of this node's connectivity and is given by

$$
\tilde{P}_{i}(t)=\frac{m}{t+m_{0}} .
$$

Similarly, the connectivity distribution and the dynamical behavior of a single node are given by [16]

$$
\begin{aligned}
\mathcal{P}(k) & =A e^{-k / m}=\frac{e}{m} e^{-k / m}, \\
k_{i}(t) & =m\left[\ln \left(m_{0}+t-1\right)-\ln \left(m_{0}+t_{i}^{0}-1\right)+1\right] \\
& =m[\ln (a t+b)+1],
\end{aligned}
$$


where $a=\frac{1}{m_{0}+t_{i}^{0}-1}, b=\frac{m_{0}-1}{m_{0}+t_{i}^{0}-1}$ and the normalization condition is $1=\int_{m}^{\infty} \mathcal{P}(k) d k$.

Substituting (9) into (7), we obtain

$$
w_{i}(t)=1+e \int_{t_{i}^{0}}^{t} \int_{m}^{\infty} \frac{1}{t^{\prime}+m_{0}} \frac{k_{i}\left(t^{\prime}\right)}{k_{i}\left(t^{\prime}\right)+k_{l}} e^{-k_{l} / m} d k_{l} d t^{\prime} .
$$

After performing the integration and inserting $k_{i}(t)$ from (9), for large $t$ we obtain

$$
w_{i}(t) \simeq m \ln (a t+b)-m \ln (\ln (a t+b)+2)+C,
$$

where $C$ is an integration constant independent of $t$. Therefore the relation between $w_{i}(t)$ and $k_{i}(t)$ for large $t$ follows

$$
w_{i}(t) \simeq k_{i}(t)-m \ln \ln t+C .
$$

The prediction (11) is fully supported by numerical simulations: in Fig. 3a we plot the difference $w_{i}(t)-k_{i}(t)$ as function of $\ln \ln (t)$, showing that the difference indeed follows a double logarithmic law. This result is very interesting since it indicates that the different slopes observed in Fig. $2 \mathrm{~b}$ for $k_{i}(t)$ and $w_{i}(t)$ do not represent distinct power law scaling behaviors, but are the result of logarithmic corrections.

WSF model: In the scale-free model the probability distributions and $k_{i}(t)$ are given by 16 .

$$
\begin{aligned}
& \tilde{P}_{i}(t)=m \frac{k_{i}(t)}{\sum_{j}^{t} k_{j}}=m \frac{k_{i}(t)}{2 m t}=\frac{k_{i}(t)}{2 t}, \\
& \mathcal{P}(k)=m k^{-2} \quad(\propto k \cdot P(k)), \\
& k_{i}(t)=\frac{m}{\sqrt{t_{i}^{0}}} \sqrt{t} .
\end{aligned}
$$

Substituting (12) into (7), and performing the integrals we obtain

$w_{i}(t) \simeq k_{i}(t)-\frac{m}{8}\left(\ln \frac{m^{2} t}{t_{i}^{0}}\right)^{2}+\frac{m}{2} \ln m \ln \frac{t}{t_{i}^{0}}+C^{\prime}$,

indicating that despite a different scaling behavior suggested by the numerical simulations (Fig. 2e), we are dealing with strong logarithmic corrections and asymptotically we have $\beta^{\prime}=\beta$. Again, the analytical prediction (13) is confirmed by more detailed numerical simulations shown in Fig. 3b.

Our ability to calculate analytically $w_{i j}$ for the discussed models is based on the fact that the weights are driven by the connectivity distribution. To address the generality of our results we investigated several extensions of these two models, that we discuss in the following.

Weight driven weight- In general one could expect that in some systems the quantity determining the weight is not the connectivity, but are the weights themselves. To investigate this possibility we replaced (2) with

$$
w_{j i}=\frac{w_{i}}{\sum_{i^{\prime}} w_{i^{\prime}}} .
$$

i.e. the weight of the newly added links are determined by the total weight of the nodes. While we cannot solve this model analytically, the numerical results are similar to those observed for the WE and WSF models: an apparently different scaling behavior for $k$ and $w$ can be attributed to slow corrections to scaling.

Weight driven connectivity- In some systems the topology could be driven by the total weights, and not by the connectivity. Thus we assume that the probability (1) that a new node is connected to a node $j$ is

$$
\Pi_{i}=\frac{w_{i}}{\sum_{j} w_{j}}
$$

where $w_{i}$ is the weight of node $i$. The weights are then assigned following (2). We find that the scaling of this network is identical to that of the scale-free model, and the evolution of the weights also follows the paradigm established for the WSF model.

Discussion- Weighted links are a common feature of real networks, thus addressing their scaling behavior is of primarily importance if we are to understand complex networks in general. Here we take a first step in this direction by investigating the scaling properties of several simple models that incorporate mechanisms to assign weights to the links. The weight distribution in the discussed models can be determined analytically and by using numerical simulations, allowing for a thorough analysis of the model's scaling properties. A comparison between the analytical and numerical results brings deeper understanding of the observed scaling behavior for the distribution functions. First, extensive simulations of networks whose size is comparable to the real networks that are currently available indicate the emergence of new scaling exponents for the behavior of the total weights. However, the analytical solutions reveal that the results are affected by strong logarithmic corrections, and asymptotically the scaling behaviors of the weighted and unweighted models are identical. This result raises important questions regarding our ability to uncover the correct scaling behavior of real weighted networks, should such data become available in the near future: the real exponents could be easily shadowed by corrections to scaling similar to that encountered in the investigated models here.

An important feature of the studied models is the fact that the distribution of the individual weights, $w_{i j}$, is bounded and stationary. This implies that the scaling behavior of the total weight distribution can not be explained by the distribution of individual weights. Indeed it is the correlation between the total weight and the linkage probability of the new node that drives the distribution towards power law behavior. In general, one can imagine systems and models where the link distribution, $P\left(w_{i j}\right)$, could also have nontrivial distribution, 
such as a power law. Investigating such models could be of major future interest.

The results presented in this paper represent only the starting point towards understanding weighted networks. In some real systems, diverse dynamical rules can govern the assignment of weights to links, which could result in statistical properties of the network that are different from that discussed here. In particular, we assumed that once a weight has been assigned to a link, it stays unchanged, which is often not the case in more realistic networks: weights can evolve dynamically just as the network topology does. For example, acquaintance can turn into friendship by strengthening a previously weak link. Determining the generic behavior of such complex evolving systems is a real challenge for future research. Despite these limitations, the investigated models give a glimpse into the complex behavior we are facing as we attempt to make network modeling more realistic by incorporating weights. Finally, the ultimate understanding of weighted networks will be determined by the available data on real systems. While currently such data is rare, we believe that the increasing interest in network modeling and creative data collection methods will soon lead to the development of such data sets, offering further guidance for modeling these complex systems.

Research at Notre Dame supported by NSF, PHY9988674 and CAREER DMR97-01998.

[1] B. Bollobás, Random Graphs, Academic, London (1985).

[2] P. Erdös and A. Rényi, Publ. Math. Inst. Hung. Acad. Sci. 5, 17 (1960).

[3] D.J. Watts, Small Worlds: The Dynamics of Networks Between Order and Randomness, (Princeton 1999).

[4] D. J. Watts and D. H. Strogatz, Nature 393, 440 (1998).

[5] M. E. J. Newman J. Stat. Phys. 101, 819 (2000).

[6] A.-L. Barabási and R. Albert, Science 286, 509 (1999).

[7] S. N. Dorogovtsev and J. F. F. Mendes Phys. Rev. E 62, 1842 (2000); S. N. Dorogovtsev and J. F. F. Mendes Europhys. Lett. 5233 (2000).

[8] P. L. Krapivsky, S. Redner, and F. Leyvraz, Phys. Rev. Lett. 85, 4629 (2000).

[9] L. A. N. Amaral, A. Scalar, M. Barthélémy, and H. E. Stanley, Proc. Nat. Acad. Sci. USA 9711149 (2000).

[10] R. Cohen, K. Erez, D. ben-Avraham, and S. Havlin, Phys. Rev. Lett. 85, 4626 (2000).

[11] M. Granovetter, Am. J. of Soc. 78, 1360 (1973).

[12] M. E. J. Newman, cond-mat/0011144

[13] E. L. Berlow, Nature 398, 330 (1999).

[14] J. R. Banavar, A. Maritan and A. Rinaldo, Nature 399,130, (1999).

[15] R. Albert, H. Jeong, and A.-L. Barabási, Nature 401, 130 (1999).

[16] A.-L. Barabási, R. Albert, and H. Jeong, Physica A 272, 173, 1(1999).
[17] L. Kullmann and J. Kertész, cond-mat/0012410.
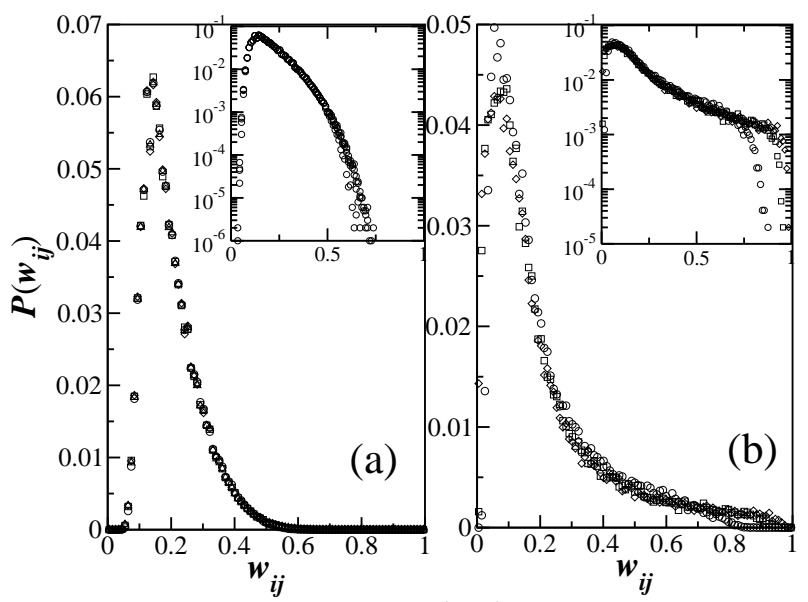

FIG. 1. The distribution $P\left(w_{i j}\right)$ of the individual link weights, $w_{i j}$ for the (a) WE and the (b) WSF models, defined in the text $(m=2)$. The symbols correspond to different system sizes (or time), i.e. $N=10^{3}(\bigcirc), 10^{4}(\square), 10^{5}(\diamond)$ and $10^{6}(\triangle)$. The insets shows the same data on a log -linear plot, indicating that the tail decays faster than exponential.
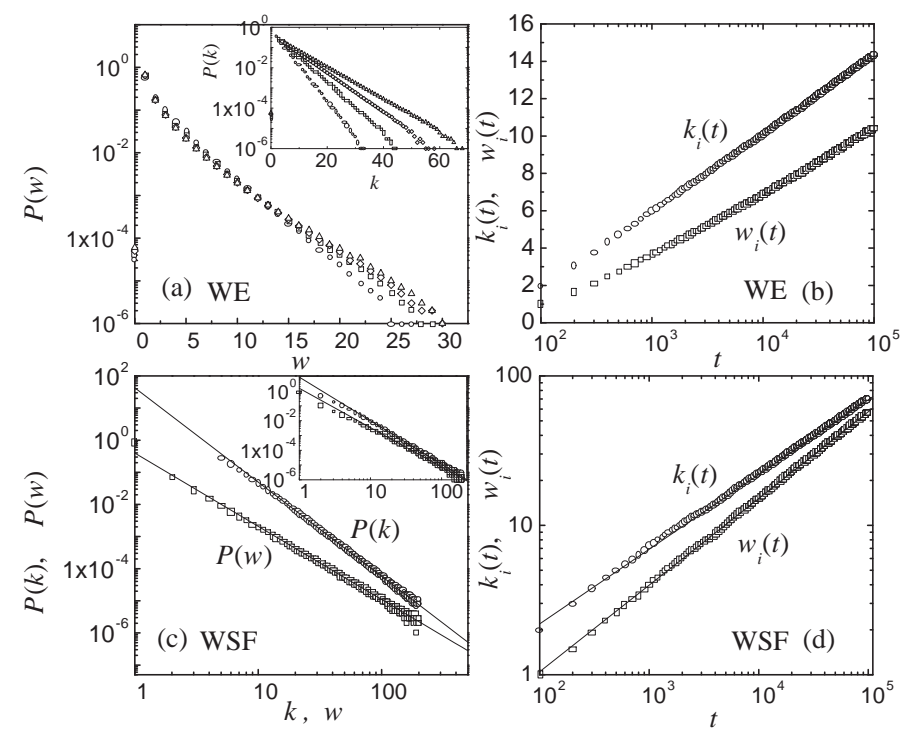

FIG. 2. (a) Distribution $P(w)$ of the total connectivity $w$ assigned to individual nodes for the WE model. The symbols correspond to different values of $m$, i.e. $m=2(\bigcirc), 3(\square)$, $4(\diamond)$ and $5(\triangle)$. The inset shows the connectivity distribution, $P(k)$, for the same parameters as in the main panel. (b) Time dependence of $k_{i}(t)(\bigcirc)$ and $w_{i}(t)(\square)$ for a randomly selected node $i$ for the WE model $(i=5000)$. (c) $P(k)(\bigcirc)$ and $P(w)(\square)$ distributions for the WSF model for $m=5$. The inset shows the same data for $m=2$. (d) $k_{i}(t)(\bigcirc)$, $w_{i}(t)(\square)$ vs. $t$ for the WSF model $(i=10000)$. 

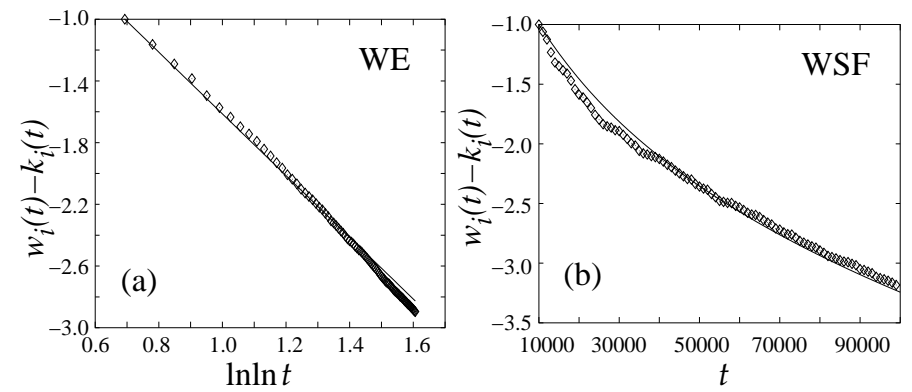

FIG. 3. The difference $\left(w_{i}(t)-k_{i}(t)\right)$ for the (a) WE and the (b) WSF models. The continuous lines in each case represent the analytic solution (11) and (13), respectively. We limited the simulations to nodes appears at large $t_{i}^{0}\left(t_{i}^{0}=10^{4}\right)$ to capture the asymptotic limit, that is predicted by our predictions (11) and (13). We find that for smaller $t_{i}^{0}$ the crossover time for the convergence to the analytic solution is numerically prohibited. 